\title{
Equivalence of measures of
} smoothness in $L_{p}\left(S^{d-1}\right), 1<p<\infty$

by

\section{F. DAi (Edmonton), Z. Ditzian (Edmonton) and Hongwei Huang (Xiamen)}

Abstract. Suppose $\widetilde{\Delta}$ is the Laplace-Beltrami operator on the sphere $S^{d-1}, \Delta_{\rho}^{k} f(x)$ $=\Delta_{\rho} \Delta_{\rho}^{k-1} f(x)$ and $\Delta_{\rho} f(x)=f(\rho x)-f(x)$ where $\rho \in S O(d)$. Then

$$
\omega^{m}(f, t)_{L_{p}\left(S^{d-1}\right)} \equiv \sup \left\{\left\|\Delta_{\rho}^{m} f\right\|_{L_{p}\left(S^{d-1}\right)}: \rho \in S O(d), \max _{x \in S^{d-1}} \rho x \cdot x \geq \cos t\right\}
$$

and

$$
\widetilde{K}_{m}\left(f, t^{m}\right)_{p} \equiv \inf \left\{\|f-g\|_{L_{p}\left(S^{d-1}\right)}+t^{m}\left\|(-\widetilde{\Delta})^{m / 2} g\right\|_{L_{p}\left(S^{d-1}\right)}: g \in \mathcal{D}\left((-\widetilde{\Delta})^{m / 2}\right)\right\}
$$

are equivalent for $1<p<\infty$. We note that for even $m$ the relation was recently investigated by the second author. The equivalence yields an extension of the results on sharp Jackson inequalities on the sphere. A new strong converse inequality for $L_{p}\left(S^{d-1}\right)$ given in this paper plays a significant role in the proof.

1. Introduction. For a Banach space $B$ of functions on the unit sphere $S^{d-1}=\left\{x \in \mathbb{R}^{d}:|x|^{2}=x_{1}^{2}+\cdots+x_{d}^{2}=1\right\}$, the $r$ th modulus of smoothness (introduced in [Di-99]) is given for $0<t<\pi / 2$ by

$$
\begin{aligned}
& \omega^{r}(f, t)_{B}=\sup \left\{\left\|\Delta_{\rho}^{r} f\right\|_{B}: \rho \in S O(d)\right. \text { and } \\
& \left.\qquad \rho x \cdot x \geq \cos t \text { for all } x \in S^{d-1}\right\}
\end{aligned}
$$

where $S O(d)$ is the group of $d \times d$ orthogonal matrices of determinant 1 , $r \in \mathbb{N}$, and $\Delta_{\rho}^{k} f(x)$ is given by

$$
\Delta_{\rho}^{k} f(x)=\Delta_{\rho}\left(\Delta_{\rho}^{k-1} f(x)\right), \quad \Delta_{\rho} f(x)=f(\rho x)-f(x) .
$$

The main result of this paper is the equivalence between $\omega^{r}(f, t)_{p}=$ $\omega^{r}(f, t)_{L_{p}\left(S^{d-1}\right)}$ and the $K$-functional $\widetilde{K}_{r}(f, t)_{p}=\widetilde{K}_{r}(f, t)_{L_{p}\left(S^{d-1}\right)}$, that is,

$$
C^{-1} \widetilde{K}_{r}\left(f, t^{r}\right)_{p} \leq \omega^{r}(f, t)_{p} \leq C \widetilde{K}_{r}\left(f, t^{r}\right)_{p}, \quad 1<p<\infty,
$$

2010 Mathematics Subject Classification: 42B15, 41A17, 41A63.

Key words and phrases: $K$-functionals, sharp Jackson inequality, realization, LaplaceBeltrami operator on the sphere. 
which we denote by $\omega^{r}(f, t)_{p} \approx \widetilde{K}_{r}\left(f, t^{r}\right)_{p}$. In this paper the symbol " $\approx$ " signifies that a relation such as (1.3) holds, and $f$ is always a real-valued function on $S^{d-1}$.

To define and describe $\widetilde{K}_{r}\left(f, t^{r}\right)_{B}$, we write

$$
\widetilde{K}_{r}\left(f, t^{r}\right)_{B}=\inf \left\{\|f-g\|_{B}+t^{r}\left\|(-\widetilde{\Delta})^{r / 2} g\right\|_{B}:(-\widetilde{\Delta})^{r / 2} g \in B\right\}
$$

where $\widetilde{\Delta}$ is the Laplace-Beltrami differential operator on $S^{d-1}$. We recall that

$$
\begin{aligned}
\widetilde{\Delta} f(\mathbf{x}) & =\Delta F(\mathbf{x}), \quad \mathbf{x} \in S^{d-1}, \quad F(\mathbf{x})=f(\mathbf{x} /|\mathbf{x}|), \\
\Delta & =\frac{\partial^{2}}{\partial x_{1}^{2}}+\cdots+\frac{\partial^{2}}{\partial x_{d}^{2}} .
\end{aligned}
$$

We now define $(-\widetilde{\Delta})^{\alpha}$ for $\alpha \in \mathbb{R}$. The finite-dimensional space $H_{k}$ of eigenfunctions of $\widetilde{\Delta}$ is given by

$$
H_{k}=\{\varphi: \widetilde{\Delta} \varphi=-k(k+d-2) \varphi\}, \quad k=0,1, \ldots,
$$

and the projection $P_{k} f$ of $f$ on $H_{k}$ is given by

$$
P_{k} f=\sum_{l=1}^{d_{k}}\left\{\int_{S^{d-1}} f(y) Y_{k, l}(y) d y\right\} Y_{k, l}
$$

where $Y_{k, l}$ is any real orthonormal basis of $H_{k}$. For any Banach space satisfying $B \subset L_{1}\left(S^{d-1}\right),(1.7)$ is well defined and the expansion of $f$ by $P_{k} f$ is denoted by $f \sim \sum P_{k} f$. The operator $(-\widetilde{\Delta})^{\alpha} f$ is then given by

$$
(-\widetilde{\Delta})^{\alpha} f \sim \sum k^{\alpha}(k+d-2)^{\alpha} P_{k} f
$$

(defined for $\alpha<0$ only when $P_{0} f=0$ ), and $(-\widetilde{\Delta})^{\alpha} f \in B$ when there exists $\Phi \in B$ such that $P_{k} \Phi=k^{\alpha}(k+d-2)^{\alpha} P_{k} f$ for all $k$. We note that for $\alpha=r \in \mathbb{N},(-\widetilde{\Delta})^{r}=(-1)^{r} \widetilde{\Delta}^{r}$ where $\widetilde{\Delta}^{r}$ is the $r$ th iterate of $\widetilde{\Delta}$ given in 1.5 .

In Section 10 we apply the equivalence 1.3 to obtain a generalization of the sharp Jackson inequalities and of the lower estimates for functions in $L_{p}\left(S^{d-1}\right), 1<p<\infty$ (see [Da-Di-Ti]), and we believe other applications will be given in the future. In Section 2 we present some additional required notations. In Sections 35 results needed to establish (1.3) for $r=1$ are given. For odd $d$, the operator 


$$
\begin{aligned}
& A_{\theta} f(x)=\int_{S O(d)} f\left(Q M_{\theta} Q^{-1} x\right) d Q, \quad \int_{S O(d)} d Q=1, \\
& M_{\theta}=\left(\begin{array}{cccccc}
\sin \theta & \cos \theta & & & & 0 \\
-\cos \theta & \sin \theta & & & & \\
& & \ddots & & & \\
0 & & & \sin \theta & \cos \theta & \\
& & & & & 1
\end{array}\right)
\end{aligned}
$$

and for combinations of $A_{j \theta} f$, a strong converse inequality of type $A$ (in the terminology of (Di-Iv] ) given in Sections 6 and 7 plays an important role in the proof of (1.3). We prove the strong converse inequality and the equivalence for a more general space than just $L_{p}\left(S^{d-1}\right)$, and we hope it will be of use in the future as well.

In Section 8 we give an alternative description of $\omega^{r}(f, t)_{B}$ by a different $K$-functional, and in Section 9 we conclude the proof of 1.3 .

2. Preliminaries. We introduce the operator

$$
D^{\alpha} f \sim \sum_{k=1}^{\infty}(2 k+d-2)^{2 \alpha} P_{k} f
$$

with $P_{k} f$ of (1.7), $\alpha \in \mathbb{R}$ and $D^{\alpha} f \in L_{p}\left(S^{d-1}\right)$ if there exists $\Phi \in L_{p}\left(S^{d-1}\right)$ such that $P_{k} \Phi=(2 k+d-2)^{2 \alpha} P_{k} f$ for all $k$. We note that $D^{\alpha} f$ is related to $(-\widetilde{\Delta})^{\alpha} f$ by

$$
\left\|(-\widetilde{\Delta})^{\alpha} f\right\|_{p} \approx\left\|D^{\alpha} f\right\|_{p} \quad \text { for } 1 \leq p \leq \infty, \alpha \in \mathbb{R} \text { and } P_{0} f=0,
$$

as can easily be verified using the Abel transformation. In fact, we have to show that $\left\{(2 k+d-2)^{2 \alpha} /(k(k+d-2))^{\alpha}\right\}$ is a bounded multiplier operator on $L_{p}\left(S^{d-1}\right)$ for all $\alpha \in \mathbb{R}$ when $P_{0} f=0$. Following [Da-Di-07, Section 4], we have to show that $\left\{\left(4+(d-2)^{2} /(k(k+d-2))\right)^{\alpha}\right\}$ satisfies the condition $E(m)$ of [Da-Di-07, Definition 4.1]. This in turn follows from the obvious estimate

$$
\left|\frac{d^{l}}{d x^{l}}\left(4+\frac{(d-2)^{2}}{x(x+d-2)}\right)^{\alpha}\right| \leq c_{l}(x+1)^{-l-1}, \quad x \geq 0, l=0,1, \ldots
$$

Note that the conditions $E(m)$ for positive and negative $\alpha$ are symmetric. We can now follow the proof of Theorem 4.3 of [Da-Di-07] and in particular of (4.7) there, which uses the Abel transformation (summation by parts). by

For $f \in C^{1}\left(S^{d-1}\right)$ we can define the tangential gradient of $f, \operatorname{grad}_{\tan } f$,

$$
\operatorname{grad}_{\tan } f=\left.\nabla F\right|_{S^{d-1}}, \quad F(\mathbf{x})=f(\mathbf{x} /|\mathbf{x}|) \quad \text { for } \mathbf{x} \in \mathbb{R} \backslash\{0\}
$$


where $\nabla F=\operatorname{grad} F$ is the gradient of $F$, i.e. $\nabla F=\left(\partial F / \partial x_{1}, \ldots, \partial F / \partial x_{d}\right)$. In terms of spherical coordinates (see [Er, Chapter XI]) given by

$$
\left\{\begin{array}{l}
x_{1}=r \cos \theta_{1} \\
x_{2}=r \sin \theta_{1} \cos \theta_{2} \\
\vdots \\
x_{d-1}=r \sin \theta_{1} \ldots \sin \theta_{d-2} \cos \varphi \\
x_{d}=r \sin \theta_{1} \ldots \sin \theta_{d-2} \sin \varphi \\
0 \leq \theta_{i} \leq \pi \text { for } 1 \leq i \leq d-2 \text { and } 0 \leq \varphi \leq 2 \pi
\end{array}\right.
$$

we have

$$
\begin{aligned}
\operatorname{grad}_{\tan } u= & \left(\frac{\partial u}{\partial \theta_{1}}, \frac{1}{\sin \theta_{1}} \frac{\partial u}{\partial \theta_{2}}, \ldots, \frac{1}{\sin \theta_{1} \ldots \sin \theta_{d-2}} \frac{\partial u}{\partial \varphi}\right) \\
\nabla u= & \left(\frac{\partial}{\partial r}, \frac{1}{r} \operatorname{grad}_{\tan } u\right), \\
\widetilde{\Delta} u= & \left(\sin \theta_{1}\right)^{-d+2} \frac{\partial}{\partial \theta_{1}}\left(\sin \theta_{1}\right)^{d-2} \frac{\partial u}{\partial \theta_{1}} \\
& +\left(\sin \theta_{1}\right)^{-2}\left(\sin \theta_{2}\right)^{-d+3} \frac{\partial}{\partial \theta_{2}}\left(\sin \theta_{2}\right)^{d-3} \frac{\partial u}{\partial \theta_{2}} \\
& +\cdots+ \\
& +\left(\sin \theta_{1} \ldots \sin \theta_{d-3}\right)^{-2}\left(\sin \theta_{d-2}\right)^{-1} \frac{\partial}{\partial \theta_{d-2}} \sin \theta_{d-2} \frac{\partial u}{\partial \theta_{d-2}} \\
& +\left(\sin \theta_{1} \ldots \sin \theta_{d-2}\right)^{-2} \frac{\partial^{2} u}{\partial \varphi^{2}}, \\
\Delta u= & r^{-d+1} \frac{\partial}{\partial r}\left(r^{d-1} \frac{\partial u}{\partial r}\right)+r^{-2} \widetilde{\Delta} u .
\end{aligned}
$$

We observe that while $\operatorname{grad}_{\tan } f$ and $\widetilde{\Delta} f$ given in (2.5) and (2.6) are not restricted to the unit sphere, they coincide on the unit sphere with (2.3) and (1.5). In particular, what may seem like a discrepancy between the number of components of (2.3) and (2.6) does not exist, as (2.5) is the projection of 2.3 on the hyperplane perpendicular to the vector $r$, i.e. $\mathbf{x} /|\mathbf{x}|$, and in the $r$ direction the derivative equals 0 . We will utilize mainly (2.3) and (1.5) but will refer to 2.5) and (2.6) which we hope will help those more familiar with those forms.

We will use many identities and estimates from [Sz] and [An-As-Ro] on Jacobi and ultraspherical polynomials and we now give the notation used there.

Definition 2.1. The Jacobi polynomials $P_{n}^{(\alpha, \beta)}(x)$ and the ultraspherical polynomials $P_{n}^{\lambda}(x)=C_{n}^{\lambda}(x)$ are orthogonal systems of polynomials with 
respect to the weights $(1-x)^{\alpha}(1+x)^{\beta}$ and $\left(1-x^{2}\right)^{\lambda-1 / 2}$ on $[-1,1]$. The normalization of $P_{n}^{(\alpha, \beta)}(x)$ and $P_{n}^{\lambda}(x)=C_{n}^{\lambda}(x)$ is given by

$$
\begin{aligned}
P_{n}^{(\alpha, \beta)}(1) & =\left(\begin{array}{c}
n+\alpha \\
n
\end{array}\right)=\frac{\Gamma(n+\alpha+1)}{n ! \Gamma(\alpha+1)} \\
P_{n}^{\lambda}(1) & =C_{n}^{\lambda}(1)=\frac{\Gamma(n+2 \lambda)}{n ! \Gamma(2 \lambda)} .
\end{aligned}
$$

The normalization (2.7) follows from [Sz, (4.1.1) and (4.3.1)] and [An-As-Ro, $\left(2.5 .13^{\prime}\right)$ ] for $P_{n}^{(\alpha, \beta)}(x)$, and from [Sz, (4.7.3), p. 81] and [An-As-Ro, p. 302] for $P_{n}^{\lambda}(x)=C_{n}^{\lambda}(x)$. Clearly, 2.7 implies

$$
P_{n}^{\lambda}(x)=C_{n}^{\lambda}(x)=\frac{\Gamma(2 \lambda+n) \Gamma(\lambda+1 / 2)}{\Gamma(2 \lambda) \Gamma(\lambda+1 / 2+n)} P_{n}^{(\lambda-1 / 2, \lambda-1 / 2)}(x) .
$$

3. The case $r=1$. For $r=1$ we obtain the equivalence as a result of the following theorem. We set $\Pi_{n}=\operatorname{span}\left(\bigcup_{k=0}^{n} H_{k}\right)$.

Theorem 3.1. Suppose $f \in \bigcup_{N=0}^{\infty} \Pi_{N}$, and $\operatorname{grad}_{\tan } f$ and $(-\widetilde{\Delta})^{\alpha} f$ are given by (2.3) and (1.8) respectively. Then for $1<p<\infty$,

$$
\left\|\operatorname{grad}_{\tan } f\right\|_{L_{p}\left(S^{d-1}\right)} \approx\left\|(-\widetilde{\Delta})^{1 / 2} f\right\|_{L_{p}\left(S^{d-1}\right)} .
$$

REMARK 3.2. The equivalence (3.1) means that whenever one side exists (i.e. is finite), the other side is finite and equivalent. The constants of the equivalence depend on $p$ and $d$ but not on $f$. In fact, we need and will prove (3.1) just for spherical harmonic polynomials, but because of the density of $\operatorname{span}\left(\bigcup_{k=0}^{\infty} H_{k}\right)$ in $L_{p}\left(S^{d-1}\right)$, (3.1) is valid whenever $(-\widetilde{\Delta})^{1 / 2} f \in L_{p}$, in which case the definition of $\operatorname{grad}_{\tan } f$ in 2.3 can be extended by density.

For the proof of Theorem 3.1 we need the following two results.

Lemma 3.3. For $f, g \in C^{2}\left(S^{d-1}\right)$,

$$
\langle-\widetilde{\Delta} f, g\rangle=\left\langle\operatorname{grad}_{\tan } f, \operatorname{grad}_{\tan } g\right\rangle \text { where }\langle\varphi, \psi\rangle \equiv \int_{S^{d-1}} \varphi(x) \psi(x) d \sigma(x) .
$$

Theorem 3.4. For $f \in \Pi_{N}, 1<p<\infty$ and $D^{\alpha} f$ given by 2.1 we have

$$
\left\|\operatorname{grad}_{\tan }\left(D^{-1 / 2} f\right)\right\|_{L_{p}\left(S^{d-1}\right)} \leq C\|f\|_{L_{p}\left(S^{d-1}\right)}
$$

where $C$ depends on $p$ and $d$ but not on $f$ and $N$.

Lemma 3.3 is known: see [Mu, p. 80, Lemma 1]. We are grateful to the referee for pointing this out. The proof of Theorem 3.4, which is the main ingredient of the proof of Theorem 3.1. will be given in Sections 4 and 5 . 
Proof of Theorem 3.1 (assuming Theorem 3.4). Using (3.3) and 2.2), we have, for $f \in \Pi_{N}$ and $1<p<\infty$,

$$
\begin{aligned}
\left\|\operatorname{grad}_{\tan } f\right\|_{p} & =\left\|\operatorname{grad}_{\tan }\left(D^{-1 / 2} D^{1 / 2} f\right)\right\|_{p} \leq C\left\|D^{1 / 2} f\right\|_{p} \\
& \leq C_{1}\left\|(-\widetilde{\Delta})^{1 / 2} f\right\|_{p} .
\end{aligned}
$$

To show the converse inequality, we assume $f \in \Pi_{N}$ and let $V_{N} f$ be an operator satisfying $V_{N} g=g$ for $g \in \Pi_{N}, V_{N} g \in \Pi_{2 N}$ for all $g \in L_{p}$ and $\left\|V_{N} g\right\|_{p} \leq A\|g\|_{p}$ for all $g \in L_{p}\left(S^{d-1}\right), 1 \leq p \leq \infty$. (That is, $V_{N}$ is a delayed mean or de la Vallée Poussin-type operator.) We now have (for $f \in \Pi_{N}$, $1<p<\infty$ and $\left.q^{-1}+p^{-1}=1\right)$

$$
\begin{aligned}
\left\|(-\widetilde{\Delta})^{1 / 2} f\right\|_{p} & =\sup _{\|g\|_{q} \leq 1}\left\langle(-\widetilde{\Delta})^{1 / 2} f, g\right\rangle \\
& =\sup _{\|g\|_{q} \leq 1}\left\langle(-\widetilde{\Delta})^{1 / 2} f, V_{N} g\right\rangle
\end{aligned}
$$

(and, as $\left\|V_{N} g\right\|_{q} \leq A\|g\|_{q}$ and $V_{N} g \in \Pi_{2 N}$ )

$$
\leq \sup _{\|h\|_{q} \leq A, h \in \Pi_{2 N}}\left\langle(-\widetilde{\Delta})^{1 / 2} f, h\right\rangle
$$

(and, as $(-\widetilde{\Delta})^{1 / 2} f$ is orthogonal to constants)

$$
\begin{aligned}
& \leq \sup _{\|h\|_{q} \leq A, h \in \Pi_{2 N}}\left\langle(-\widetilde{\Delta})^{1 / 2} f, h-P_{0} h\right\rangle \\
& \leq \sup _{\|h\|_{q} \leq A_{1}, h \in \Pi_{2 N}, P_{0} h=0}\left\langle(-\widetilde{\Delta})^{1 / 2} f, h\right\rangle \\
& =\sup _{\|h\|_{q} \leq A_{1}, P_{0} h=0, h \in \Pi_{2 N}}\left\langle(-\widetilde{\Delta})^{1 / 2} f,(-\widetilde{\Delta})^{1 / 2}\left((-\widetilde{\Delta})^{-1 / 2} h\right)\right\rangle \\
& =\sup _{\|h\|_{q} \leq A_{1}, P_{0} h=0, h \in \Pi_{2 N}}\left\langle(-\widetilde{\Delta}) f,(-\widetilde{\Delta})^{-1 / 2} h\right\rangle
\end{aligned}
$$

(which, recalling Lemma 3.3)

$$
=\sup _{\|h\|_{q} \leq A_{1}, P_{0} h=0, h \in \Pi_{2 N}}\left\langle\operatorname{grad}_{\tan } f, \operatorname{grad}_{\tan }\left((-\widetilde{\Delta})^{-1 / 2} h\right)\right\rangle
$$

(and by the Hölder inequality)

$$
\leq \sup _{\|h\|_{q} \leq A_{1}, P_{0} h=0, h \in \Pi_{2 N}}\left\|\operatorname{grad}_{\tan } f\right\|_{p}\left\|\operatorname{grad}_{\tan }(-\widetilde{\Delta})^{-1 / 2} h\right\|_{q}
$$

(and by Theorem 3.4)

$$
\leq C\left\|\operatorname{grad}_{\tan } f\right\|_{p} \sup _{\|h\|_{q} \leq A_{1}, h \in \Pi_{2 N}}\left\|D^{1 / 2}(-\widetilde{\Delta})^{-1 / 2} h\right\|_{q}
$$


(and by (2.2p)

$$
\leq C_{1}\left\|\operatorname{grad}_{\tan } f\right\|_{p} \sup _{\|h\|_{q} \leq A_{1}}\|h\|_{q} \leq C_{1} A_{1}\left\|\operatorname{grad}_{\tan } f\right\|_{p}
$$

4. Essential estimates. In this section we will prove several estimates that will culminate in the proof of Theorem 3.4 in the next section.

For $P_{k}^{((d-3) / 2,(d-3) / 2)}$ given in Definition 2.1 we have

$$
P_{k} f(x)=A(k, d) \int_{S^{d-1}} f(y) P_{k}^{((d-3) / 2,(d-3) / 2)}(x \cdot y) d \sigma(y)
$$

where $d \sigma(y)$ is the Haar measure on $S^{d-1}$ satisfying $\int_{S^{d-1}} d \sigma(y)=\left|S^{d-1}\right|$, and

$$
A(k, d)=\frac{1}{\left|S^{d-1}\right|} \frac{(d+2 k-2) \Gamma((d-1) / 2)(k+d-3) !}{(d-2) ! \Gamma(k+(d-1) / 2)}
$$

as follows from [St-We, pp. 143-144] together with (2.7). We define the multiple of $P_{k}^{(\alpha, \beta)}(x), E_{k}^{(\alpha, \beta)}(x)$, by

$$
\begin{aligned}
E_{k}^{(\alpha, \beta)}(t)= & \frac{\Gamma((d-1) / 2)}{\Gamma(d-1)} \frac{1}{\left|S^{d-1}\right|} \\
& \times \frac{(2 k+\alpha+\beta+1) \Gamma(k+\alpha+\beta+1)}{\Gamma(k+\beta+1)} P_{k}^{(\alpha, \beta)}(t),
\end{aligned}
$$

and by [St-We, pp. 143-144], 4.1) takes the form

$$
P_{k} f(x)=\int_{S^{d-1}} f(y) E_{k}^{((d-3) / 2,(d-3) / 2)}(x \cdot y) d y .
$$

We now define the kernel $K_{N}(t)$ by

$$
K_{N}(t) \equiv \sum_{k=1}^{2 N} \eta\left(\frac{k}{N}\right) \frac{1}{2 k+d-2} E_{k}^{((d-3) / 2,(d-3) / 2)}(t)
$$

where $\eta \in C^{\infty}[0, \infty), \eta(t)=1$ for $t \leq 1$ and $\eta(t)=0$ for $t \geq 2$.

We need the estimate of $K_{N}^{(i)}(\cos \theta)$ given in the following result.

Theorem 4.1. For $i=0,1, \ldots$ and $\theta \in(0, \pi)$,

$$
\left|K_{N}^{(i)}(\cos \theta)\right| \leq C\left(N^{-1}+\theta\right)^{-d-2 i+2}
$$

where $K_{N}^{(i)}(\cos \theta)$ is $K_{N}^{(i)}(t)=(d / d t)^{i} K_{N}(t)$ evaluated at $t=\cos \theta$.

For the proof of (4.4) we need the following lemma.

Lemma 4.2. For $\alpha \geq \beta \geq 0$ and $k=0,1, \ldots$ we have

$$
\frac{1}{2 k+\alpha+\beta+2} E_{k}^{(\alpha+1, \beta)}(t)=\sum_{j=0}^{k} E_{j}^{(\alpha, \beta)}(t)
$$


and

(4.6) $\quad\left|E_{k}^{(\alpha, \beta)}(\cos \theta)\right|$

$$
\leq \begin{cases}C k^{2 \alpha+1}, & 0 \leq \theta \leq k^{-1} \\ C k^{\alpha+1 / 2} \theta^{-\alpha-1 / 2}(\pi-\theta)^{-\beta-1 / 2}, & k^{-1} \leq \theta \leq \pi-k^{-1} \\ C k^{\alpha+\beta+1}, & \pi-k^{-1}<\theta \leq \pi\end{cases}
$$

Proof. The identity 4.5 follows from [Sz, (9.4.3), p. 255], in which we use $k=0$ and recall 4.2 and $C_{l}^{(k)}=\left(\begin{array}{c}l+k \\ l\end{array}\right)$. The estimate 4.6 follows from [Sz, (7.32.5) and (4.1.3)] using (4.2) and

$$
\Gamma(x+a) / \Gamma(x)=x^{a}+O\left(x^{a-1}\right) \quad \text { as } x \rightarrow \infty \text { for } a \in \mathbb{R} .
$$

We are now in a position to prove Theorem 4.1.

Proof of Theorem 4.1. We follow the proof of Lemma 3.1 of Br-Da. Using [Sz, (4.21.7), p. 63] we have

$$
\frac{d}{d t}\left\{P_{k}^{(\alpha, \beta)}(t)\right\}=\frac{k+\alpha+\beta+1}{2} P_{k-1}^{(\alpha+1, \beta+1)}(t)
$$

and hence

$$
\frac{d}{d t}\left\{E_{k}^{(\alpha, \beta)}(t)\right\}=\frac{1}{2} E_{k-1}^{(\alpha+1, \beta+1)}(t) .
$$

Therefore,

$$
\begin{aligned}
& \left(\frac{d}{d t}\right)^{i} K_{N}(t) \equiv K_{N}^{(i)}(t) \\
& \quad=C(i) \sum_{k=0}^{2 N-i} \eta\left(\frac{k+i}{N}\right) \frac{1}{2 k+2 i+d-2} E_{k}^{((d-3) / 2+i,(d-3) / 2+i)}(t),
\end{aligned}
$$

which, by Lemma 4.2 , implies

$$
\left|K_{N}^{(i)}(t)\right| \leq C_{1}(i)\left(1+\sum_{k=1}^{2 N-i} k^{d-3+2 i}\right) \leq C_{2}(i) N^{d-2+2 i}
$$

Using 4.5, we have

$$
\overleftarrow{\Delta}\left\{\frac{1}{2 k+\alpha+\beta+2} E_{k}^{(\alpha+1, \beta)}(t)\right\}=E_{k}^{(\alpha, \beta)}(t)
$$

where $E_{-1}^{(\alpha+1, \beta)}(t)=0, \overleftarrow{\Delta} b_{k+1}=b_{k+1}-b_{k}$ and $\vec{\Delta} c_{k}=c_{k}-c_{k+1}$ 
Therefore, using the Abel transformation (summation by parts), we have $(4.10)$

$$
\begin{aligned}
K_{N}^{(i)}(t)= & C(i) \sum_{k=0}^{\infty} \eta\left(\frac{k+i}{N}\right) \frac{1}{2 k+2 i+d-2} \\
& \times \overleftarrow{\Delta}\left\{\frac{1}{2 k+2 i+1+d-2} E_{k}^{((d-3) / 2+i+1,(d-3) / 2+i)}(t)\right\} \\
= & C(i) \sum_{k=0}^{\infty} \vec{\Delta}\left\{\eta\left(\frac{k+i}{N}\right) \frac{1}{2 k+2 i+d-2}\right\} \\
& \times \frac{1}{2 k+2 i+1+d-2} E_{k}^{((d-3) / 2+i+1,(d-3) / 2+i)}(t) \\
= & C(i) \sum_{k=0}^{\infty} a_{N, j}(k) \frac{1}{2 k+d-2+2 i+j} E_{k}^{((d-3) / 2+i+j,(d-3) / 2+i)}(t)
\end{aligned}
$$

where $a_{N, 0}(k)=\eta\left(\frac{k+i}{N}\right)$ and $a_{N, j+1}(k) \equiv \vec{\Delta}\left\{\frac{a_{N, j}(k)}{2 k+d-2+2 i+j}\right\}$.

We now observe that, for $a_{r} \geq 1$,

$$
\left|(\vec{\Delta})^{m} \frac{1}{k+a_{r}}\right| \leq \frac{m !}{(k+1)^{m+1}} .
$$

Furthermore, as $\eta(u) \in C^{\infty}\left(\mathbb{R}_{+}\right)$and supp $\eta(u) \subset[0,2)$, one has

$$
\left|(\vec{\Delta})^{l} \eta\left(\frac{k+i}{N}\right)\right| \leq \frac{A_{l}}{N^{l}} \quad \text { and hence }\left|(\vec{\Delta})^{l} \eta\left(\frac{k+i}{N}\right)\right| \leq \frac{2^{l}}{(k+1)^{l}} .
$$

As $a_{N, j}(k)$ is a finite sum of terms which are constant times

$$
(\vec{\Delta})^{m_{1}} \eta\left(\frac{k+i}{N}\right) \prod_{r=2}^{j+1}(\vec{\Delta})^{m_{r}} \frac{1}{k+a_{r}}
$$

where $a_{r} \geq 1, m_{1}+\cdots+m_{j+1}=j$ and $m_{r} \geq 0$, we have $\left|a_{N, j}(k)\right| \leq$ $C /(k+1)^{2 j}$.

Choosing $j$ such that $j>d / 2+i-1$ and using (4.6) for $\theta \in\left(N^{-1}, \pi / 2\right)$, we now have

$$
\begin{aligned}
\left|K_{N}^{(i)}(\cos \theta)\right| & \leq C(j, i)\left(1+\sum_{1 \leq k \leq \theta^{-1}} k^{d-3+2 i}+\theta^{-d / 2+1-i-j} \sum_{\theta^{-1} \leq k} k^{d / 2+i-j-2}\right) \\
& \equiv C(j, i)(1+I(\theta)+J(\theta)) .
\end{aligned}
$$

As $I(\theta) \leq C_{1} \theta^{-d+2-2 i}$, we have, for $N \theta \geq 1$,

$$
I(\theta) \leq C_{1} \theta^{-d+2-2 i} \leq C_{1}\left(N^{-1}+\theta\right)^{-d-2 i+2} .
$$

Recalling that $j>d / 2+i-1$, we have

$$
J(\theta) \leq C_{2} \theta^{-d / 2+1-i-j} \leq C_{2} \theta^{-d-2 i+2} \leq C_{2}\left(N^{-1}+\theta\right)^{-d-2 i+2} .
$$


For $\theta \in[\pi / 2, \pi]$ we choose $j \geq d-3+2 i+2$, and using (4.6) and (4.10), we obtain

$$
\left|K_{N}^{(i)}(\cos \theta)\right| \leq C_{2} \sum_{k=1}^{2 N-i} k^{-2 j-1} k^{d-3+2 i+j+1}=C_{2} \sum_{k=1}^{2 N-i} k^{d-3+2 i-j} \leq C_{3} .
$$

Combining the above estimates with (4.8) (for the case $\theta \in\left[0, N^{-1}\right]$ ), we conclude the proof of (4.4).

For $x \cdot y=\cos \theta, \theta \in[0, \pi]$, the Euclidean distance $|x-y|$ satisfies $|x-y|=2\left|\sin \frac{\theta}{2}\right| \approx|\theta|=\arccos x \cdot y$, and we have the following corollary of Theorem 4.1

Corollary 4.3. Define

$$
G_{j}(x, y)=K_{N}^{\prime}(x \cdot y)\left[y_{j}-(y \cdot x) x_{j}\right], \quad 1 \leq j \leq d .
$$

Then for $x, y, z \in S^{d-1}$,

(1) $\left|G_{j}(x, y)\right| \leq C /\left(N^{-1}+|x-y|\right)^{d-1}$,

(2) $|y-z| \leq \frac{1}{2}\left(N^{-1}+|x-y|\right)$ implies

$$
\left|G_{j}(x, y)-G_{j}(x, z)\right| \leq \frac{C|y-z|}{\left(N^{-1}+|x-y|\right)^{d}},
$$

(3) $|x-z| \leq \frac{1}{2}\left(N^{-1}+|x-y|\right)$ implies

$$
\left|G_{j}(x, y)-G_{j}(z, y)\right| \leq \frac{C|x-z|}{\left(N^{-1}+|x-y|\right)^{d}} .
$$

Proof. Using (4.4) with $i=1$ and $\left|y_{j}-(y \cdot x) x_{j}\right| \leq|y-(y \cdot x) x|=|\sin \theta| \leq$ $2\left|\sin \frac{\theta}{2}\right|=|x-y|$, where $\theta=\arccos x \cdot y$, we obtain (1). The condition $|y-z| \leq$ $\frac{1}{2}\left(N^{-1}+|x-y|\right)$ implies $|x-z|+N^{-1} \leq \frac{3}{2}\left(|x-y|+N^{-1}\right) \leq 3\left(|x-z|+N^{-1}\right)$ or $|x-z|+N^{-1} \approx|x-y|+N^{-1}$. We have

$$
\left|K_{N}^{\prime}(x \cdot y)-K_{N}^{\prime}(x \cdot z)\right|=\left|K_{N}^{\prime \prime}\left(\cos \theta_{1}\right)\right||x \cdot y-x \cdot z|=I
$$

where $\cos \theta_{1}$ is between $x \cdot y$ and $x \cdot z$ and where $\theta_{1} \in[0, \pi]$. Using (4.4) with $i=2$, we have

$$
\left|K_{N}^{\prime \prime}\left(\cos \theta_{1}\right)\right| \leq C\left(N^{-1}+\theta_{1}\right)^{-d-2} \leq C_{1}\left(N^{-1}+|x-y|\right)^{-d-2} .
$$

Furthermore,

$$
\begin{aligned}
|x \cdot y-x \cdot z| & =\frac{1}{2}|| x-\left.y\right|^{2}-|x-z|^{2} \mid \\
& \leq \frac{1}{2}|| x-y|-| x-z||(|x-y|+|x-z|) \\
& \leq \frac{5}{4}|y-z|\left(|x-y|+N^{-1}\right) .
\end{aligned}
$$

Therefore,

$$
I \leq C_{2}\left(N^{-1}+|x-y|\right)^{-d-1}|y-z| .
$$


We now use (4.4) to estimate $\left|K_{N}^{\prime}(x \cdot y)\right|$, and as

$$
\left|y_{j}-(y \cdot x) x_{j}-z_{j}+(x \cdot z) x_{j}\right| \leq|(y-z)-((y-z) \cdot x) x| \leq|y-z|,
$$

we complete the proof of $(2)$.

The proof of (3) is the same when $x$ and $y$ are interchanged.

5. Proof of Theorem 3.4. We are now ready to prove Theorem 3.4 . which is in fact a crucial step in proving Theorem 3.1. To do this, we need the following Calderón-Zygmund decomposition, which is a special case of the result given in [St, p. 17 and p. 37, 8.1] in the context of the sphere.

Theorem 5.1 (Calderón-Zygmund decomposition, Stein). Suppose $f \in$ $L_{1}\left(S^{d-1}\right)$ and $\alpha>\left|S^{d-1}\right|^{-1} \int_{S^{d-1}}|f(y)| d \sigma(y)$. Then there exists a decomposition $f=g+b$ with $b=\sum_{k} b_{k}$ and a sequence $\left\{B_{k}\right\}$ of spherical caps so that:

I. $|g(x)| \leq C \alpha$ for a.e. $x \in S^{d-1}$,

II. $\operatorname{supp} b_{k}(x) \subset B_{k},\left|B_{k}\right|^{-1} \int_{B_{k}}\left|b_{k}(x)\right| d \sigma(x) \leq c \alpha$ and $\int_{S^{d-1}} b_{k}(x) d \sigma(x)$ $=0$

III. $\sum_{k}\left|B_{k}\right| \leq(C / \alpha) \int_{S^{d-1}}|f(x)| d \sigma(x)$.

We note that a spherical cap is the intersection of $S^{d-1}$ with a ball in $\mathbb{R}^{d}$ whose center is in $S^{d-1}$.

Now we are in a position to prove Theorem 3.4 .

Proof of Theorem 3.4. We assume $f \in \Pi_{N}$. By definition, that is, using (2.1) and (4.3), we have

$$
D^{-1 / 2} f(x)=\int_{S^{d-1}} f(y) K_{N}(x \cdot y) d \sigma(y)
$$

Therefore,

$$
\begin{aligned}
\operatorname{grad}_{\tan }\left(D^{-1 / 2} f(x)\right) & =\int_{S^{d-1}} f(y) \operatorname{grad}_{\tan }\left[K_{N}(\langle\cdot, y\rangle)\right](x) d \sigma(y) \\
& =\int_{S^{d-1}} f(y) K_{N}^{\prime}(x \cdot y)(y-(x \cdot y) x) d \sigma(y) \\
& =\left(T_{1} f(x), \ldots, T_{d} f(x)\right)
\end{aligned}
$$

where $\langle\cdot, \cdot\rangle$ denotes the usual dot product in $\mathbb{R}^{d}$, and

$$
T_{j} f(x)=\int_{S^{d-1}} f(y) G_{j}(x, y) d \sigma(y)
$$

with $G_{j}(x, y)$ given by 4.11. To prove our theorem, it suffices to show

$$
\left\|T_{j} f\right\|_{p} \leq C\|f\|_{p} \quad \text { for } j=1, \ldots, d, 1<p<\infty
$$


with $C$ independent of $N$. For $p=2$ we use Lemma 3.3 to obtain

$$
\begin{aligned}
\left\|T_{j} f\right\|_{2}^{2} & \leq\left\|\operatorname{grad}_{\tan }\left(D^{-1 / 2} f\right)\right\|_{2}^{2}=\left\|(-\widetilde{\Delta})^{1 / 2}\left(D^{-1 / 2} f\right)\right\|_{2}^{2} \\
& \leq\left\|D^{1 / 2}\left(D^{-1 / 2} f\right)\right\|_{2}^{2} \leq\|f\|_{2}^{2} .
\end{aligned}
$$

To prove (5.2) for $1<p \leq 2$ we may use the Marcinkiewicz interpolation theorem (see [St-We, p. 184, Theorem 2.4]) and show that $T_{j} f$ is of weak type $(1,1)$, that is,

$$
|E(\alpha, j)| \equiv\left|\left\{x \in S^{d-1}:\left|T_{j}(f)(x)\right|>2 C \alpha\right\}\right| \leq C_{1} \frac{\|f\|_{1}}{\alpha} .
$$

As $E(\alpha, j) \subset S^{d-1},|E(\alpha, j)| \leq\left|S^{d-1}\right|$, and hence for $\alpha \leq\|f\|_{1}$, (5.5) is valid with $C_{1} \geq\left|S^{d-1}\right|$. It remains to prove (5.5) for $\alpha>\|f\|_{1}$, for which Theorem 5.1 is applicable. For $\alpha>\|f\|_{1}$ we assume $f=g+b$ with $g$ and $b$ of Theorem 5.1. Applying (5.4) to $g$ gives

$$
\left|\left\{x \in S^{d-1}:\left|T_{j} g(x)\right|>C \alpha\right\}\right| \leq C^{-2} \frac{\left\|T_{j} g\right\|_{2}^{2}}{\alpha^{2}} \leq C^{-2} \frac{\|g\|_{2}^{2}}{\alpha^{2}} \leq C^{\prime} \frac{\|g\|_{1}}{\alpha} .
$$

Using I and III of Theorem 5.1, we have

$$
\int_{S^{d-1} \backslash \cup B_{k}}|g(x)| d \sigma(x)=\int_{S^{d-1} \backslash \cup B_{k}}|f(x)| d \sigma(x) \leq \int_{S^{d-1}}|f(x)| d \sigma(x)
$$

and

$$
\int_{\bigcup B_{k}}|g(x)| d \sigma(x) \leq C \alpha\left|\bigcup B_{k}\right| \leq C \alpha \sum\left|B_{k}\right| \leq C^{2} \int_{S^{d-1}}|f(x)| d \sigma(x) .
$$

Hence $C^{\prime}\|g\|_{1} / \alpha \leq C_{1}\|f\|_{1} / \alpha$.

For each $k$ we assume $B_{k}=B\left(x_{k}, r_{k}\right) \cap S^{d-1}$ with $x_{k} \in S^{d-1}$ and $B_{k}^{*}=$ $B\left(x_{k}, 4 r_{k}\right) \cap S^{d-1}$. For $x \notin B_{k}^{*}\left(x \in S^{d-1}\right)$, using II of Theorem 5.1, we have

$$
\begin{aligned}
\left|T_{j}\left(b_{k}\right)(x)\right| & =\left|\int_{B_{k}}\left(G_{j}(x, y)-G_{j}\left(x, x_{k}\right)\right) b_{k}(y) d \sigma(y)\right| \\
& \leq C^{\prime} \int_{B_{k}} \frac{\left|y-x_{k}\right|}{\left|x-x_{k}\right|^{d}}\left|b_{k}(y)\right| d \sigma(y) \leq C^{\prime} \frac{r_{k}}{\left|x-x_{k}\right|^{d}} \int_{B_{k}}\left|b_{k}(y)\right| d \sigma(y) \\
& \leq C_{1}^{\prime}\left|B_{k}\right| \alpha \frac{r_{k}}{\left|x-x_{k}\right|^{d}},
\end{aligned}
$$

and hence

$$
\int_{\left(B_{k}^{*}\right)^{c}}\left|T_{j}\left(b_{k}\right)(x)\right| d \sigma(x) \leq C_{2}^{\prime} \alpha\left|B_{k}\right| .
$$

Therefore, 


$$
\begin{aligned}
\mid\left\{x \in S^{d-1}:\left|T_{j} b(x)\right|\right. & >C \alpha\} \mid \\
& \leq\left|\bigcup_{k} B_{k}^{*}\right|+\left|\left\{x \in\left(\bigcup_{k} B_{k}^{*}\right)^{c}:\left|T_{j}(b)(x)\right| \geq C \alpha\right\}\right| \\
& \leq \sum_{k}\left|B_{k}^{*}\right|+\frac{1}{C \alpha} \int_{\left(\bigcup_{k} B_{k}^{*}\right)^{c}}\left|T_{j}(b)(x)\right| d \sigma(x) \\
& \leq \sum_{k}\left|B_{k}^{*}\right|+\frac{1}{C \alpha} \sum_{k} \int_{\left(B_{k}^{*}\right)^{c}}\left|T_{j}\left(b_{k}\right)\right| d \sigma(x) \\
& \leq C_{3} \sum_{k}\left|B_{k}\right| \leq C_{4} \frac{\|f\|_{1}}{\alpha} .
\end{aligned}
$$

This concludes the proof of $(5.3)$ for $1<p \leq 2$.

To prove 5.3 for $2<p<\infty$, we define

$$
T_{j}^{*} g(x)=\int_{S^{d-1}} f(y) G_{j}(y, x) d \sigma(y) .
$$

Using Fubini's theorem, we have

$$
\int_{S^{d-1}} T_{j} f(x) \cdot g(x) d \sigma(x)=\int_{S^{d-1}} f(x) T_{j}^{*} g(x) d \sigma(x),
$$

and hence $\left\|T_{j}^{*}\right\|_{\left(p^{\prime}, p^{\prime}\right)}=\left\|T_{j}\right\|_{(p, p)}$. We now follow the considerations earlier in the proof of Theorem 3.4 using (3) of Corollary 4.3 (instead of (2)), to show

$$
\left\|T_{j}^{*} g\right\|_{q} \leq C^{\prime}\|g\|_{q} \text { for } 1<q \leq 2,
$$

which, together with $(5.6)$, completes the proof of $(5.3)$ and of our theorem.

6. A strong converse inequality of type $A$. We define the operator $A_{\theta}$ by

$$
A_{\theta} f(x)=\int_{S O(d)} f\left(Q^{-1} M_{\theta} Q x\right) d Q, \quad \int_{S O(d)} d Q=1,
$$

where $Q \in S O(d), x \in S^{d-1}, d Q$ is the Haar measure on $S O(d)$ and $M_{\theta}$ is the $d \times d$ matrix given by

$$
M_{\theta}=\left[\begin{array}{ccc}
\cos \theta \sin \theta & & \\
-\sin \theta \cos \theta & & \\
& \ddots & \\
& & \cos \theta \sin \theta \\
& & -\sin \theta \cos \theta
\end{array}\right] \text { or }\left[\begin{array}{cccc}
\cos \theta \sin \theta & & \\
-\sin \theta \cos \theta & & \\
& \ddots & & \\
& & \cos \theta \sin \theta & \\
& -\sin \theta \cos \theta & \\
& & & 1
\end{array}\right]
$$


when $d$ is even or odd respectively. Clearly, $A_{\theta}$ is a contraction operator on $L_{p}\left(S^{d-1}\right), 1 \leq p \leq \infty$. In fact, on a Banach space $B$ of functions $f$ on $S^{d-1}$ satisfying

$$
\|f(\rho \cdot)\|_{B}=\|f(\cdot)\|_{B} \quad \text { and } \quad\|f(\rho \cdot)-f(\cdot)\|_{B} \rightarrow 0 \quad \text { as }|\rho-I| \rightarrow 0
$$

where $\rho \in S O(d)$ and $|\rho-I|=\max _{x \in S^{d-1}}|\rho x-x|, A_{\theta}$ is also a well-defined continuous contraction operator, that is, satisfying $\left\|A_{\theta} f\right\|_{B} \leq\|f\|_{B}$ and $\left\|A_{\theta_{1}} f-A_{\theta} f\right\|_{B}=o(1)$ as $\left|\theta_{1}-\theta\right| \rightarrow 0$.

The main result of this section, which is proved in the next section, is the following strong converse inequality of type $A$ (in the terminology of [Di-Iv]), which yields an equivalence between special combinations of $A_{j \theta} f$ and the appropriate $K$-functionals.

Theorem 6.1. Suppose $l \in N, 0<\theta \leq \pi /(2 l), f \in B \subset L_{1}\left(S^{d-1}\right)$ for some $d \geq 3$, and the Banach space $B$ satisfies $(6.3)$. Then

$$
\left\|A_{\theta, l} f-f\right\|_{B} \approx \inf _{\widetilde{\Delta}^{l} g \in B}\left(\|f-g\|_{B}+\theta^{2 l}\left\|\widetilde{\Delta}^{l} g\right\|_{B}\right) \equiv \widetilde{K}_{2 l}\left(f, \theta^{2 l}\right)_{B}
$$

where

$$
A_{\theta, l} f(x)=\frac{-2}{\left(\begin{array}{c}
2 l \\
l
\end{array}\right)} \sum_{j=1}^{l}(-1)^{j}\left(\begin{array}{c}
2 l \\
l-j
\end{array}\right) A_{j \theta} f(x)
$$

and $A_{\tau} f(x)$ is given in (6.1).

Remark 6.2. For even dimension $d(d>3)$,

$$
A_{\theta} f(x)=S_{\theta} f(x)=\frac{1}{m_{\theta}} \int_{x \cdot y=\cos \theta} f(y) d y, \quad m_{\theta}=\int_{x \cdot y=\cos \theta} d y
$$

(see [Da-Di-08, Th. 2.1]), and hence $A_{\theta, l} f=S_{\theta, l} f$ with $S_{\theta, l} f$ of Da-Di-04, (1.7)]. This means that Theorem 6.1 was already proved (for even $d$ ) in [Da-Di-04, Th. 4.1] for $B=L_{p}\left(S^{d-1}\right)(1 \leq p \leq \infty)$ and in Da-Di-08, Th. 3.3] for $B$ satisfying (6.3).

For the proof of Theorem 6.1 (for odd $d$ ) we need a few lemmas. As we use many results from $[\mathrm{Sz}]$ and from $[\mathrm{An}-\mathrm{As}-\mathrm{Ro}]$, we remind the reader again that the systems of polynomials $\left\{P_{n}^{(\alpha, \beta)}(x)\right\}$ and $\left\{C_{n}^{\lambda}(x)\right\}=\left\{P_{n}^{\lambda}(x)\right\}$ are the orthogonal polynomials with respect to $(1-x)^{\alpha}(1+x)^{\beta}$ and $\left(1-x^{2}\right)^{\lambda-1 / 2}$ on $[-1,1]$ which are normalized by (2.7) (see Definition 2.1).

Perhaps the crucial result of this section is the simple description of $A_{\theta} f$ (for odd $d$ ) given in the following theorem. 
Theorem 6.3. Suppose $A_{\theta} f$ is given by (6.1) with odd $d \geq 3$ and $M_{\theta}$ is given in 6.2. Then for $f \in L_{1}\left(S^{d-1}\right)$ satisfying $f \sim \sum_{k=0}^{\infty} P_{k} f$ we have

$$
A_{\theta} f \sim \sum_{n=0}^{\infty} R_{n}^{(\lambda, \lambda-1)}(\cos \theta) P_{n} f, \quad \lambda=\frac{d-2}{2}, \quad R_{n}^{(\lambda, \lambda-1)}(x)=\frac{P_{n}^{(\lambda, \lambda-1)}(x)}{P_{n}^{(\lambda, \lambda-1)}(1)} .
$$

For comparison, we note that Da-Di-08, Th. 2.1] and classical results imply, for even $d>3$,

$$
A_{\theta} f \sim \sum_{n=0}^{\infty} R_{n}^{(\lambda, \lambda)}(\cos \theta) P_{n} f, \quad \lambda=\frac{d-2}{2}, \quad R_{n}^{(\lambda, \lambda)}(x)=\frac{P_{n}^{(\lambda, \lambda)}(x)}{P_{n}^{(\lambda, \lambda)}(1)} .
$$

Proof. Using [Da-Di-08, Th. 4.1, (4.4)], we have

$$
A_{\theta} f(x)=c \int_{0}^{\pi / 2} \cos ^{d-2} \varphi S_{\psi(\varphi, \theta)} f(x) d \varphi
$$

where $c \int_{0}^{\pi / 2} \cos ^{d-2} \varphi d \varphi=1$ and $\sin \left(\frac{1}{2} \psi(\varphi, \theta)\right)=\left(\sin \frac{\theta}{2}\right) \cos \varphi$. Since we have $S_{\psi(\varphi, \theta)} h(x) \in H_{n}$ for $h \in H_{n}$, it follows that

$$
P_{n}\left(A_{\theta} f\right)=c \int_{0}^{\pi / 2} \cos ^{d-2} \varphi S_{\psi(\varphi, \theta)} P_{n} f d \varphi
$$

and hence

$$
A_{\theta} f \sim \sum_{n=0}^{\infty} m_{n}(\theta) P_{n} f, \quad m_{n}(\theta)=c \int_{0}^{\pi / 2} \cos ^{d-2} \varphi \frac{C_{n}^{\lambda}(\cos \psi(\varphi, \theta))}{C_{n}^{\lambda}(1)} d \varphi .
$$

A change of variables and use of the definition of $\psi(\varphi, \theta)$ yield

$$
m_{n}(\theta)=\frac{c}{2 C_{n}^{\lambda}(1)} \int_{0}^{\pi / 2} \sin ^{2 \lambda} \varphi C_{n}^{\lambda}\left(\cos ^{2} \varphi+\sin ^{2} \varphi \cos \theta\right) d \varphi .
$$

Thus, using (2.8) and the fact that $m_{n}(0)=1$, we are reduced to showing

$$
R_{n}^{(\lambda, \lambda-1)}(\cos \theta)=c \int_{0}^{\pi / 2} \sin ^{2 \lambda} \varphi R_{n}^{(\lambda-1 / 2, \lambda-1 / 2)}\left(\cos ^{2} \varphi+\sin ^{2} \varphi \cos \theta\right) d \varphi
$$

where $R_{n}^{(\alpha, \beta)}(t)=P_{n}^{(\alpha, \beta)}(t) / P_{n}^{(\alpha, \beta)}(1)$. The referee kindly pointed out that (6.8) can be deduced easily from the following formula which can be found in [Sz, (4.10.11), p. 96]:

$$
\begin{aligned}
& (1-x)^{\alpha+\mu} R_{n}^{(\alpha+\mu, \beta-\mu)}(x) \\
& \quad=\frac{\Gamma(\alpha+\mu+1)}{\Gamma(\alpha+1) \Gamma(\mu)} \int_{x}^{1}(1-y)^{\alpha} R_{n}^{(\alpha, \beta)}(y)(y-x)^{\mu-1} d y .
\end{aligned}
$$


In fact, performing a change of variable $y=(1-\cos \theta) \cos ^{2} \varphi+\cos \theta$, we deduce from 6.9 that

$$
\begin{aligned}
& R_{n}^{(\alpha+\mu, \beta-\mu)}(\cos \theta) \\
& \quad=\frac{\Gamma(\alpha+\mu+1)}{\Gamma(\alpha+1) \Gamma(\mu)}(1-\cos \theta)^{-\alpha-\mu} \int_{\cos \theta}^{1}(1-y)^{\alpha} R_{k}^{(\alpha, \beta)}(y)(y-\cos \theta)^{\mu-1} d y \\
& \quad=\frac{2 \Gamma(\alpha+\mu+1)}{\Gamma(\alpha+1) \Gamma(\mu)} \int_{0}^{\pi / 2} \sin ^{2 \alpha+1} \varphi \cos ^{2 \mu-1} \varphi R_{n}^{(\alpha, \beta)}\left(\cos ^{2} \varphi+\sin ^{2} \varphi \cos \theta\right) d \varphi .
\end{aligned}
$$

Now setting $\alpha=\beta=\lambda-1 / 2$ and $\mu=1 / 2$, we obtain the desired equation (6.8). This completes the proof.

To prove Theorem 6.1 for $B$ satisfying 6.3 and not just for $L_{p}$, $1 \leq p<\infty$, or when $d$ is even, we need the following lemma.

Lemma 6.4. For $B$ satisfying 6.3), $B \subset L_{1}\left(S^{d-1}\right)$ and $\delta>d-1$ one has

$$
\left\|C_{N}^{\delta} f\right\|_{B} \leq C\|f\|_{B}
$$

where

$$
C_{N}^{\delta} f=\sum_{k=0}^{N} \frac{A_{N-k}^{\delta}}{A_{N}^{\delta}} P_{k} f \quad \text { and } \quad A_{j}^{\delta}=\frac{\Gamma(j+\delta+1)}{\Gamma(j+1) \Gamma(\delta+1)} .
$$

Proof. We define

$$
K_{N}^{\delta}(\cos \theta)=\sum_{k=0}^{N} \frac{A_{N-k}^{\delta}}{A_{N}^{\delta}}\left\|R_{k}^{(\lambda, \lambda-1)}\right\|_{2, w_{\lambda}}^{-2} R_{k}^{(\lambda, \lambda-1)}(\cos \theta)
$$

with $\|g\|_{p, w_{\lambda}}=\left\{\int_{0}^{\pi}|g(\theta)|^{p} w_{\lambda}(\theta) d \theta\right\}^{1 / p}$ and

$$
w(\theta)=w_{\lambda}(\theta)=\left(\sin \frac{\theta}{2}\right)^{2 \lambda+1}\left(\cos \frac{\theta}{2}\right)^{2 \lambda-1} .
$$

Using [Bo-Cl, Th. 2.1, pp. 230-231] for $\delta>2 \lambda+1=d-1$, we have

$$
\left|K_{N}^{\delta}(\cos \theta)\right| \leq C N^{2 \lambda+2}(1+N \theta)^{-2 \lambda-3} \quad \text { for } \theta \in[0, \pi]
$$

and hence

$$
\sup _{N} \int_{0}^{\pi}\left|K_{N}(\cos \theta)\right| w_{\lambda}(\theta) d \theta \leq C_{1}
$$

It is sufficient to show the identity

$$
C_{N}^{\delta}(f)=\int_{0}^{\pi} A_{\theta} f K_{N}^{\delta}(\cos \theta) w_{\lambda}(\theta) d \theta
$$


since, as $A_{\theta} f$ is continuous and a contraction in $B$, it will imply $\left\|C_{N}^{\delta} f\right\|_{B} \leq$ $C_{1}\|f\|_{B}$. To prove the above identity, we observe that, for $0 \leq k \leq N$,

$$
\begin{aligned}
P_{k}\left(\int_{0}^{\pi} A_{\theta} f K_{N}^{\delta}(\cos \theta) w_{\lambda}(\theta) d \theta\right) & =\left[\int_{0}^{\pi} R_{k}^{(\lambda, \lambda-1)}(\cos \theta) K_{N}^{\delta}(\cos \theta) w_{\lambda}(\theta) d \theta\right] P_{k} f \\
& =\frac{A_{N-k}^{\delta}}{A_{N}^{\delta}} P_{k} f=P_{k}\left(C_{N}^{\delta} f\right) .
\end{aligned}
$$

For convenience we state the following well-known elementary multiplier theorem which is an immediate result of the Abel transformation (summation by parts) and $P_{k} f=(\overleftarrow{\Delta})^{m+1}\left\{\left(\begin{array}{c}k+m \\ m\end{array}\right) C_{k}^{m} f\right\}$ where $\overleftarrow{\Delta} \mu(k)=$ $\mu(k)-\mu(k-1),(\overleftarrow{\Delta})^{r} \mu(k)=\overleftarrow{\Delta}\left((\overleftarrow{\Delta})^{r-1} \mu(k) g\right), C_{k}^{m} f=0$ for $k<0$ and $C_{k}^{m} f$ is given by 6.10) with $m=\delta$ for $k \geq 0$.

TheOREM 6.5. Suppose

$$
\left\|C_{n}^{m} f\right\|_{B} \leq C\|f\|_{B}, \quad \sum_{k=0}^{\infty}\left(\begin{array}{c}
k+m \\
k
\end{array}\right)\left|(\vec{\Delta})^{m+1} \mu(k)\right|<C
$$

and $\mu(k)=o(1)$ as $k \rightarrow \infty$. Then

$$
\left\|T_{\mu} f\right\|_{B} \leq C_{1}\|f\|_{B} \quad \text { where } \quad T_{\mu} f \sim \sum_{k=0}^{\infty} \mu(k) P_{k} f .
$$

7. Proof of Theorem 6.1. In Section 6 we stated the strong converse inequality, i.e. Theorem 6.1, and established the setup and preliminary results needed for its proof, which we now give.

Proof of Theorem 6.1. As noted in Remark 6.2, we need to prove 6.4 only for odd $d \geq 3$. For the delayed means $\eta_{a \theta} f$ given by

$$
\eta_{a \theta} f=\sum_{k=0}^{\infty} \eta(a \theta k) P_{k} f, \quad \eta \in C^{\infty}\left(\mathbb{R}_{+}\right), \quad \eta(x)= \begin{cases}1, & 0 \leq x \leq 1, \\ 0, & 2 \leq x,\end{cases}
$$

we have the realization result

$$
\widetilde{K}_{2 r}\left(f, \theta^{2 r}\right)_{B} \approx\left\|f-\eta_{a \theta} f\right\|_{B}+\theta^{2 r}\left\|(-\widetilde{\Delta})^{r} \eta_{a \theta} f\right\|_{B}
$$

(see [Di-98, Th. 7.1] in which 7.2 is proved using (6.10) for any delayed means). To prove (6.4) we follow [Da-Di-04, Th. 4.1], and using (7.2), it is enough to show for some $a>0$ that

$$
\begin{aligned}
\left\|f-A_{l, \theta} f\right\|_{B} & \geq C\left\|f-\eta_{a \theta} f\right\|_{B}, \\
\left\|f-A_{l, \theta} f\right\|_{B} & \geq C \theta^{2 l}\left\|(-\widetilde{\Delta})^{l} \eta_{a \theta} f\right\|_{B}, \\
\left\|\eta_{a \theta} f-A_{l, \theta} \eta_{a \theta} f\right\|_{B} & \leq C \theta^{2 l}\left\|\widetilde{\Delta}^{l} \eta_{a \theta} f\right\|_{B},
\end{aligned}
$$


which correspond to [Da-Di-04, (5.3)-(5.5)]. It seems now that here and in [Da-Di-04, Section 5] it is sufficient to deal with $a=1$, but in order not to repeat and marginally modify the calculations in [Da-Di-04, we maintain $\eta_{a \theta}$.

We may also follow [Da-Di-04, (5.6)] and prove as an alternative to 7.3 that

$$
\left\|A_{l, \theta}^{5}\left(f-\eta_{a \theta} f\right)\right\|_{B} \leq C\left\|f-A_{l, \theta} f\right\|_{B},
$$

since $\left\|A_{l, \theta} f\right\|_{B} \leq 2^{l}\|f\|_{B}$ and $\left\|\eta_{a \theta} f\right\|_{B} \leq C_{1}\|f\|_{B}$ imply

$$
\begin{aligned}
\left\|\left(I-A_{l, \theta}^{5}\right)\left(f-\eta_{a \theta} f\right)\right\|_{B} & =\left\|\left(I+A_{l, \theta}+\cdots+A_{l, \theta}^{4}\right)\left(I-A_{l, \theta}\right)\left(f-\eta_{a \theta} f\right)\right\|_{B} \\
& \leq C_{2}\left\|f-A_{l, \theta} f\right\|_{B} .
\end{aligned}
$$

We now set

$$
a_{l}(k, \theta)=\frac{-2}{\left(\begin{array}{c}
2 l \\
l
\end{array}\right)} \sum_{j=1}^{l}(-1)^{j}\left(\begin{array}{c}
2 l \\
l-j
\end{array}\right) R_{k}^{(\lambda, \lambda-1)}(\cos j \theta),
$$

and using Lemma 6.4 and Theorem 6.1, we only have to show that the moments

$$
\begin{aligned}
\mu_{k}^{(1)} & =\frac{1-\eta(a \theta k)}{1-a_{l}(k, \theta)} a_{l}(k, \theta)^{5}, \\
\mu_{k}^{(2)} & =\frac{\left(k(k+2 \lambda) \theta^{2}\right)^{l}}{1-a_{l}(k, \theta)} \eta(a \theta k), \\
\mu_{k}^{(3)} & =\frac{1-a_{l}(k, \theta)}{\left(k(k+2 \lambda) \theta^{2}\right)^{l}} \eta(a \theta k)
\end{aligned}
$$

satisfy the conditions of Theorem 6.5 for some $m>d-1(m>2 \lambda+1)$.

To do this, we first show that for nonnegative integers $s, k$ and $\theta \in$ $[0, \pi / 2)$, we have

$$
\left|(\vec{\Delta})^{s} R_{k}^{(\alpha, \beta)}(\cos \theta)\right| \leq C(s, \alpha, \beta) \theta^{s}(1+k \theta)^{-\alpha-1 / 2},
$$

where $R_{k}^{(\alpha, \beta)}(t)=P_{k}^{(\alpha, \beta)}(t) / P_{k}^{(\alpha, \beta)}(1)$.

We use [An-As-Ro, (6.4.20), p. 304] together with (6.7) to obtain

$$
\begin{aligned}
\vec{\Delta} R_{k}^{(\alpha, \beta)}(\cos \theta) & =R_{k}^{(\alpha, \beta)}(\cos \theta)-R_{k+1}^{(\alpha, \beta)}(\cos \theta) \\
& =(1-\cos \theta) \frac{2 k+\alpha+\beta+2}{2(\alpha+1)} R_{k}^{(\alpha+1, \beta)}(\cos \theta),
\end{aligned}
$$

and by induction

$$
\left|(\vec{\Delta})^{s} R_{k}^{(\alpha, \beta)}(\cos \theta)\right| \leq C_{1}(s, \alpha, \beta) \max _{2 i \leq s}(1-\cos \theta)^{s-i} k^{s-2 i}\left|R_{k}^{(\alpha+s-i, \beta)}(\cos \theta)\right|,
$$

which implies (7.7) using [Sz, (8.21.18), p. 196] for $k \theta \geq 1$, and using $\left|R_{k}^{(\alpha+s, \beta)}(\cos \theta)\right| \leq 1$ otherwise. 
We observe that for the estimate of $\mu_{k}^{(1)}$ we need for $k \geq 1 /(a \theta)$ the inequalities $1-a_{l}(k, \theta) \geq v_{\alpha, l}>0$ and $0<C_{1} \leq\left(1-a_{l}(k, \theta)\right) /(k \theta)^{2 l}<$ $C_{2}<\infty$ for $\theta \in[0, \pi / 2]$, both of which follow from [Sz, $(4.7 .29)$, p. 83] which yields

$$
\begin{aligned}
R_{k}^{(\lambda, \lambda-1)}(\cos \theta)= & \frac{1}{C_{k}^{\lambda+1 / 2}(1)+C_{k-1}^{\lambda+1 / 2}(1)} C_{k}^{\lambda+1 / 2}(\cos \theta) \\
& +\frac{1}{C_{k-1}^{\lambda+1 / 2}(1)+C_{k}^{\lambda+1 / 2}(1)} C_{k-1}^{\lambda+1 / 2}(\cos \theta)
\end{aligned}
$$

and the result on $C_{k}^{\lambda+1 / 2}(\cos \theta)$ given in [Da-Di-04, Th. 4.4, p. 278].

The proof now follows the proof in [Da-Di-04, pp. 281-283], with 7.7 used instead of Lemma 4.3 of [Da-Di-04].

8. An equivalence relation. In this section we will introduce and establish a $K$-functional equivalent to $\omega^{r}(f, t)_{p}=\omega^{r}(f, t)_{L_{p}\left(S^{d-1}\right)}, 1 \leq p \leq \infty$. This will not be $\widetilde{K}_{r}\left(f, t^{r}\right)_{p}$, but will be of help in proving $\widetilde{K}_{r}\left(f, t^{r}\right)_{p} \approx$ $\omega^{r}(f, t)_{p}$ for $1<p<\infty$. We believe that the equivalence proved in this section (Theorem 8.2 is of independent interest and will turn out useful for various purposes in the future.

Definition 8.1. A $d \times d$ skew-symmetric matrix $M$ is of class $\mathcal{M}$ if

(8.1) $\quad M=\left(\begin{array}{ccccccc}0 & \alpha_{1} & & & & \\ -\alpha_{1} & 0 & & & & & \\ & & \ddots & & & \\ & & & 0 & \alpha_{k} & \\ & & & -\alpha_{k} & 0 & & \\ & & & & & \ddots & \\ & & & & & & 0\end{array}\right), \quad 0<\alpha_{k} \leq \cdots \leq \alpha_{1} \leq 1$.

It is known (see [Di-99, p. 191] or [Ga, pp. 287-288]) that $\rho \in S O(d)$ satisfies $|\rho-I| \leq 2 \sin \frac{\theta}{2}$ (or $\rho x \cdot x \geq \cos \theta$ for all $x \in S^{d-1}$ ) if and only if it can be represented as $\rho=e^{\theta Q M Q^{-1}}$ with $Q \in S O(d)$ and $M \in \mathcal{M}$.

For $g(x) \in C^{r}\left(S^{d-1}\right),\left.(\partial / \partial t)^{r} g\left(e^{t Q M Q^{-1}} x\right)\right|_{t=u}$ exists in $C\left(S^{d-1}\right)$, that is, for each fixed $u$ the derivative is in $C\left(S^{d-1}\right)$ and is continuous in $u$ for $u \in \mathbb{R}$. We may consider $\left.(\partial / \partial t)^{r} g\left(e^{t Q M Q^{-1}} x\right)\right|_{t=u}$ as an element of $L_{p}\left(S^{d-1}\right)$, $1 \leq p \leq \infty$, and denote it by $g^{(r)}\left(e^{u Q M Q^{-1}} \cdot\right)$. In fact, for $g \in C^{r}\left(S^{d-1}\right)$ and any space of functions $B \supset C\left(S^{d-1}\right)$ which satisfies $(6.3), g^{(r)}\left(e^{u Q M Q^{-1}} \cdot\right)$ is 
defined and satisfies

$$
\begin{aligned}
& \left\|g^{(r)}\left(e^{u_{1} Q M Q^{-1}} \cdot\right)\right\|_{B}=\left\|g^{(r)}\left(e^{u_{2} Q M Q^{-1}} \cdot\right)\right\|_{B}, \\
& \left\|g^{(r)}\left(e^{u_{1} Q M Q^{-1}} \cdot\right)-g^{(r)}\left(e^{u_{2} Q M Q^{-1}} \cdot\right)\right\|_{B} \rightarrow 0 \quad \text { as } u_{1}-u_{2} \rightarrow 0 .
\end{aligned}
$$

For $P_{n} \in \operatorname{span}\left(\bigcup_{k=0}^{n-1} H_{k}\right)$ we have $P_{n}^{(r)}\left(e^{u Q M Q^{-1}} \cdot\right) \in \operatorname{span}\left(\bigcup_{k=0}^{n-1} H_{k}\right)$, since when $\varphi \in H_{k}$, the quotient $\left[\varphi\left(e^{(u+t) Q M Q^{-1}} x\right)-\varphi\left(e^{u Q M Q^{-1}} x\right)\right] / t$ is in $H_{k}$, and so is its limit (in $C\left(S^{d-1}\right), L_{p}\left(S^{d-1}\right)$ or $B$ satisfying 6.3) as $t \rightarrow 0$.

We now define the $K$-functional $K_{r}\left(f, t^{r}\right)_{B}$ and the realization functional $R_{r}\left(f, n^{-r}\right)_{B}$ for $B$ satisfying (6.3) and $C\left(S^{d-1}\right) \subset B \subset L_{1}\left(S^{d-1}\right)$ by

$$
\begin{aligned}
K_{r}\left(f, t^{r}\right)_{B}=\inf \left\{\|f-g\|_{B}+t^{r} \sup _{\substack{M \in \mathcal{M} \\
Q \in S O(d)}}\left\|g^{(r)}\left(e^{u Q M Q^{-1}} \cdot\right)\right\|_{B}:\right. \\
\left.g \in C^{r}\left(S^{d-1}\right)\right\}
\end{aligned}
$$

and

$$
R_{r}\left(f, n^{-r}\right)_{B}=\left\|f-P_{n}\right\|_{B}+n^{-r} \sup _{\substack{M \in \mathcal{M} \\ Q \in S O(d)}}\left\|P_{n}^{(r)}\left(e^{u Q M Q^{-1}} \cdot\right)\right\|_{B}
$$

with $P_{n} \in \operatorname{span}\left(\bigcup_{k=0}^{n-1} H_{k}\right)$ and

$$
\left\|f-P_{n}\right\|_{B}=E_{n}(f)_{B} \equiv \inf \left\{\|f-\varphi\|_{B}: \varphi \in \operatorname{span}\left(\bigcup_{k=0}^{n-1} H_{k}\right)\right\} .
$$

The main result of this section is now given in the following theorem.

Theorem 8.2. Suppose that $f \in L_{p}\left(S^{d-1}\right)$ with $1 \leq p<\infty$, or $f \in$ $C\left(S^{d-1}\right)$ for $p=\infty$. Then

$$
\omega^{r}(f, t)_{L_{p}\left(S^{d-1}\right)} \approx K_{r}\left(f, t^{r}\right)_{L_{p}\left(S^{d-1}\right)} \approx R_{r}\left(f,[1 / t]^{-r}\right)_{L_{p}\left(S^{d-1}\right)}
$$

where $K_{r}\left(f, t^{r}\right)_{L_{p}\left(S^{d-1}\right)}$ and $R_{r}\left(f,[1 / t]^{-r}\right)_{L_{p}\left(S^{d-1}\right)}$ are given by 8.3 and (8.4) for $B=L_{p}\left(S^{d-1}\right)$.

We note that the concepts in (8.3), (8.4) and 8.5 are given for a Banach space more general than $L_{p}\left(S^{d-1}\right)$ for the benefit of Remark 8.3 .

Proof. For $g \in C^{r}\left(S^{d-1}\right)$ and $\rho$ satisfying $\rho x \cdot x \geq \cos t$ (for all $x \in S^{d-1}$ ) we have, for $\rho=\exp \left(t Q M Q^{-1}\right)$ with $M \in \mathcal{M}$,

$$
\Delta_{\rho}^{r} g(\cdot)=\int_{0}^{t} \cdots \int_{0}^{t} g^{(r)}\left(\exp \left(\left(u_{1}+\cdots+u_{r}\right) Q M Q^{-1}\right) \cdot\right) d u_{1} \ldots d u_{r} .
$$

Using (8.2) for $B$ satisfying $B \subset C\left(S^{d-1}\right)$ and (6.3), and in particular for $B=L_{p}\left(S^{d-1}\right), 1 \leq p<\infty$, or $B=C\left(S^{d-1}\right)$, we have

$$
\left\|\Delta_{\rho}^{r} g(\cdot)\right\|_{B} \leq t^{r}\left\|g^{(r)}\left(e^{u Q M Q^{-1}} \cdot\right)\right\|_{B}
$$


Choosing now $g$ close to the infimum in 8.3 , writing $f=f-g+g$ and recalling $\left\|\Delta_{\rho}^{r}(f-g)\right\|_{B} \leq 2^{r}\|f-g\|_{B}$, we have

$$
\omega^{r}\left(f, t^{r}\right)_{B} \leq(1+\varepsilon) 2^{r} K_{r}\left(f, t^{r}\right)_{B} .
$$

Furthermore, using the definitions 8.3 and $(8.4)$, we have

$$
K_{r}\left(f, t^{r}\right)_{B} \leq R_{r}\left(f,[1 / t]^{-r}\right)_{B}
$$

In view of the Jackson inequality (see [Di-04]), we now need only show that

$$
n^{-r}\left\|P_{n}^{(r)}\left(e^{u Q M Q^{-1}} \cdot\right)\right\|_{L_{p}\left(S^{d-1}\right)} \leq C_{1} \omega^{r}(f, 1 / n)_{L_{p}\left(S^{d-1}\right)}
$$

for $P_{n}$ satisfying 8.5 with $B=L_{p}\left(S^{d-1}\right)$ to establish $R_{r}\left(f, n^{-1}\right)_{L_{p}\left(S^{d-1}\right)} \leq$ $C_{2} \omega^{r}(f, 1 / n)_{L_{p}\left(S^{d-1}\right)}$ and complete the proof of (8.6). To prove (8.7) we need the Bernstein-type inequality

$$
\left\|P_{n}^{(r)}\left(e^{u Q M Q^{-1}} \cdot\right)\right\|_{L_{p}\left(S^{d-1}\right)} \leq C_{3} n^{r}\left\|P_{n}(\cdot)\right\|_{L_{p}\left(S^{d-1}\right)}
$$

for any $P_{n} \in \operatorname{span}\left(\bigcup_{k=0}^{n-1} H_{k}\right)$. As the derivative of $P_{n}\left(e^{\tau Q M Q^{-1}} \cdot\right)$ with respect to $\tau$ is in $\operatorname{span}\left(\bigcup_{k=0}^{n-1} H_{k}\right)$, it is sufficient to prove $(8.8)$ for $r=1$ and use that as the first and inductive step in proving 8.8 ,

To prove 8.8 for $r=1$ we observe that

$$
\left\|P_{n}^{\prime}\left(e^{u Q M Q^{-1}} \cdot\right)\right\|_{L_{p}\left(S^{d-1}\right)} \leq\left\|\operatorname{grad}_{\tan } P_{n}(\cdot)\right\|_{L_{p}\left(S^{d-1}\right)} .
$$

We now follow [Di-04, Section 9] (used also in Section 4 here) to write

$$
\begin{aligned}
G_{n}(x \cdot y) & =\sum_{k=0}^{2 n} \eta\left(\frac{k}{n}\right) \sum_{l=1}^{d_{k}} Y_{k, l}(x) Y_{k, l}(y) \\
& =\sum_{k=0}^{2 n} \eta\left(\frac{k}{n}\right) E_{k}^{((d-3) / 2,(d-3) / 2)}(x \cdot y)
\end{aligned}
$$

with $Y_{k, l}(x)$ any orthonormal basis of $H_{k}, \eta(t)$ as given for 4.3), and $E_{k}^{((d-3) / 2,(d-3) / 2)}(t)$ given in 4.2 . We now have

$$
P_{n}(x)=\int_{S^{d-1}} P_{n}(y) G_{n}(x \cdot y) d y
$$

and

$$
\begin{aligned}
\left|\operatorname{grad}_{\tan } P_{n}(x)\right| & =\left|\int_{S^{d-1}} P_{n}(y) \operatorname{grad}_{\tan }\left[G_{n}(\langle\cdot, y\rangle)\right](x) d y\right| \\
& \leq \int_{S^{d-1}}\left|P_{n}(y) G_{n}^{\prime}(x \cdot y)\right| \sqrt{1-(x \cdot y)^{2}} d y .
\end{aligned}
$$


Therefore,

$$
\begin{aligned}
& \left\|\operatorname{grad}_{\tan } P_{n}\right\|_{L_{p}\left(S^{d-1}\right)} \\
& \quad \leq C(d)\left\|P_{n}\right\|_{L_{p}\left(S^{d-1}\right)} \cdot\left\|G_{n}^{\prime}(t)\left(1-t^{2}\right)^{(d-2) / 2}\right\|_{L_{1}[-1,1]}
\end{aligned}
$$

where

$$
C(d)\left\|G_{n}(t)\left(1-t^{2}\right)^{(d-3) / 2}\right\|_{L_{p}[-1,1]} \leq \int_{S^{d-1}}\left|G_{n}(x \cdot y)\right| d y \leq M .
$$

Using the Bernstein inequality for the polynomials $G_{n}(t)$ (of degree $2 n$ ) with weight $\left(1-t^{2}\right)^{(d-2) / 2}$ in $L_{1}[-1,1]$ (see [Di-To, Th. 8.4.7]), we have

$$
\begin{aligned}
\left\|\operatorname{grad}_{\tan } P_{n}\right\|_{L_{p}\left(S^{d-1}\right)} & \leq \widetilde{C} n\left\|P_{n}\right\|_{L_{p}\left(S^{d-1}\right)} \int_{S^{d-1}}\left|G_{n}(x \cdot y)\right| d y \\
& \leq \widetilde{C}_{1} n\left\|P_{n}\right\|_{L_{p}\left(S^{d-1}\right)},
\end{aligned}
$$

and hence 8.8 ).

To prove (8.7) we first prove the weaker inequality

$$
\left\|P_{n}^{(r+1)}\left(e^{u Q M Q^{-1}} \cdot\right)\right\|_{L_{p}\left(S^{d-1}\right)} \leq C_{2} n^{r+1} \omega^{r}(f, 1 / n)_{L_{p}\left(S^{d-1}\right)} .
$$

We write

$$
P_{n}=P_{n}-P_{2^{l}}+\sum_{j=1}^{l}\left(P_{2^{j}}-P_{2^{j-1}}\right)+P_{1} \quad \text { where } \quad l=\max \left\{j: 2^{j}<n\right\}
$$

and use (8.8) with $r+1$ (instead of $r$ ) and with $n$ or $2^{j}$ (instead of just $n$ ) to obtain

$$
\begin{aligned}
\left\|P_{n}^{(r+1)}\left(e^{u Q M Q^{-1}} \cdot\right)\right\|_{L_{p}\left(S^{d-1}\right)} & \leq M \sum_{j=1}^{l} 2^{j(r+1)} E_{2^{j}}(f)_{p} \\
& \leq M_{1} \sum_{j=1}^{l} 2^{j(r+1)} \omega^{r}\left(f, 2^{-j}\right)_{p} \\
& \leq M_{2} \sum_{j=1}^{l} 2^{j(r+1)} 2^{(l-j) r} \omega^{r}\left(f, 2^{-l}\right)_{p} \\
& \leq C_{2} n^{r+1} \omega^{r}\left(f, n^{-1}\right)_{p},
\end{aligned}
$$

thus establishing (8.12).

Since $\left\|f-P_{n}\right\|_{B} \leq C_{3} \omega^{r}(f, 1 / n)_{B}$ was proved for $B=L_{p}\left(S^{d-1}\right)$ in Di-04] and for more general $B$ in [Da-Di-08, Th. 6.3], we obtain $\omega^{r}\left(P_{n}, 1 / n\right)_{L_{p}\left(S^{d-1}\right)}$ $\leq\left(2^{r} C_{3}+1\right) \omega^{r}(f, 1 / n)_{p}$. We now write 


$$
\begin{aligned}
& \left\|n^{-r} P_{n}^{(r)}\left(e^{u Q M Q^{-1}} \cdot\right)-\Delta_{\exp \left(n^{-1} Q M Q^{-1}\right)}^{r} P_{n}\left(e^{u Q M Q^{-1}} \cdot\right)\right\|_{L_{p}\left(S^{d-1}\right)} \\
& \quad=\left\|\int_{0}^{1 / n} \cdots \int_{0}^{1 / n} \int_{0}^{u_{1}+\cdots+u_{r}} P_{n}^{(r+1)}\left(\exp (u+t) Q M Q^{-1} \cdot\right) d t d u_{1} \ldots d u_{r}\right\|_{L_{p}\left(S^{d-1}\right)} \\
& \quad \leq r n^{-r-1}\left\|P_{n}^{(r+1)}\left(\exp \left(v Q M Q^{-1} \cdot\right)\right)\right\|_{L_{p}\left(S^{d-1}\right)} \leq r C_{2} \omega^{r}\left(f, n^{-1}\right)_{p} .
\end{aligned}
$$

Therefore,

$$
\begin{aligned}
\left\|n^{-r} P_{n}^{(r)}\left(e^{u Q M Q^{-1}} \cdot\right)\right\|_{L_{p}\left(S^{d-1}\right)} & \leq r C_{2} \omega^{r}\left(f, n^{-1}\right)_{p}+\left(2^{r} C_{3}+1\right) \omega^{r}\left(f, n^{-1}\right)_{p} \\
& \leq C_{4} \omega^{r}\left(f, n^{-1}\right)_{p} .
\end{aligned}
$$

REMARK 8.3. Examining the proof of Theorem 8.2, one can deduce 8.6 for any Orlicz space $O$ satisfying $(6.3)$ and $C\left(S^{d-1}\right) \subset O \subset L_{1}\left(S^{d-1}\right)$, and some other spaces. As we conjecture and hope that $(8.6)$ will be valid for any Banach space $B$ of functions on $S^{d-1}$ satisfying (6.3) and $C\left(S^{d-1}\right) \subset$ $B \subset L_{1}\left(S^{d-1}\right)$, which will follow from $(8.8)$ for such spaces, we concentrated here on the $L_{p}\left(S^{d-1}\right)$ case which is needed for the proof of the main theorem of this paper.

9. The main result. In this section we will prove the equivalence 1.3 written also as

$$
\omega^{r}(f, t)_{p} \approx \widetilde{K}_{r}\left(f, t^{r}\right)_{p}, \quad 1<p<\infty, r=1,2, \ldots,
$$

which is the main result of this paper. We first prove:

Theorem 9.1. For a function space $B$ on $S^{d-1}(d \geq 3)$ satisfying $C\left(S^{d-1}\right) \subset B \subset L_{1}\left(S^{d-1}\right)$ and $(6.3)$, we have

$$
\widetilde{K}_{2 m}\left(f, \theta^{2 m}\right)_{B} \leq C \omega^{2 m}(f, \theta)_{B}, \quad m=1,2, \ldots
$$

Proof. Using $M_{\theta}$ of 6.2 (which depends on whether $d$ is even or odd), we have

$$
\begin{aligned}
\omega^{2 m}(f, \theta)_{B} & \geq \sup \left\{\left\|\Delta_{\rho}^{2 m} f\right\|_{B}: \rho=Q M_{\theta} Q^{-1}, Q \in S O(d)\right\} \\
& \geq \int_{Q \in S O(d)}\left\|\Delta_{Q M_{\theta} Q^{-1}}^{2 m} f\right\|_{B} d Q .
\end{aligned}
$$

Since $T(\rho) f(x)=f(\rho x)$ satisfies $\|T(\rho) f\|_{B}=\|f\|_{B}$, we obtain

$$
\begin{aligned}
\omega^{2 m}(f, \theta)_{B} & \geq \int_{Q \in S O(d)}\left\|T\left(\left(Q M_{\theta} Q^{-1}\right)^{m}\right) \Delta_{Q M_{\theta} Q^{-1}}^{2 m} f\right\|_{B} d Q \\
& \geq\left\|\int_{Q \in S O(d)} T\left(\left(Q M_{\theta} Q^{-1}\right)^{m}\right) \Delta_{Q M_{\theta} Q^{-1}}^{2 m} f d Q\right\|_{B} \\
& =\left(\begin{array}{c}
2 m \\
m
\end{array}\right)\left\|A_{\theta, m} f-f\right\|_{B} \geq C^{-1} \widetilde{K}_{2 m}\left(f, \theta^{2 m}\right)_{B},
\end{aligned}
$$

where in the last step we used Theorem 6.1. 
For odd $m$ we have only the following somewhat weaker result.

Theorem 9.2. For $1<p<\infty, d \geq 3$ and $m=1,2, \ldots$,

$$
\widetilde{K}_{m}\left(f, \theta^{m}\right)_{p} \leq C \omega^{m}(f, \theta)_{p} .
$$

Proof. For even $m,(9.3)$ was already proved in Theorem 9.1, and hence we only have to prove $(9.3)$ for $m=2 l-1$ and $l=1,2, \ldots$ For $P_{n}$ satisfying $\left\|f-P_{n}\right\|_{p}=E_{n}(f)_{p}, P_{n} \in \operatorname{span}\left(\bigcup_{k=0}^{n-1} H_{k}\right)$, and using the definition of $\widetilde{K}_{m}\left(f, \theta^{m}\right)_{p}$ and a Jackson-type inequality (see [Di-04, Th. 8.1]), we have

$$
\begin{aligned}
\widetilde{K}_{m}\left(f, n^{-m}\right)_{p} & \leq\left\|f-P_{n}\right\|_{p}+n^{-m}\left\|(-\widetilde{\Delta})^{m / 2} P_{n}\right\|_{p} \\
& \leq C_{1} \omega^{r}\left(f, n^{-1}\right)_{p}+n^{-m}\left\|(-\widetilde{\Delta})^{m / 2} P_{n}\right\|_{p} .
\end{aligned}
$$

Since $\omega^{r}\left(P_{n}, n^{-1}\right)_{p} \leq \omega^{r}\left(P_{n}-f, n^{-1}\right)_{p}+\omega^{r}\left(f, n^{-1}\right)_{p} \leq\left(C_{1} 2^{r}+1\right) \omega^{r}\left(f, n^{-1}\right)_{p}$, and as both $(-\widetilde{\Delta})^{m / 2}$ and $\omega^{r}$ annihilate constants, it is sufficient to show

$$
n^{-m}\left\|(-\widetilde{\Delta})^{m / 2} P_{n}\right\|_{p} \leq C_{2} \omega^{m}\left(P_{n}, n^{-1}\right)_{p}
$$

for any $P_{n} \in \operatorname{span}\left(\bigcup_{k=0}^{n-1} H_{k}\right)$.

For $m=2 l-1$ we apply Theorem 9.1 to $(-\widetilde{\Delta})^{1 / 2} P_{n}$ and the realization result (see [Di-98, Th. 8.1]) to obtain

$$
\begin{aligned}
\omega^{2 l}\left((-\widetilde{\Delta})^{-1 / 2} P_{n}, n^{-1}\right)_{p} & \geq C^{-1} \widetilde{K}_{2 l}\left((-\widetilde{\Delta})^{-1 / 2} P_{n}, n^{-2 l}\right)_{p} \\
& \geq C_{1}^{-1} n^{-2 l}\left\|(-\widetilde{\Delta})^{l-1 / 2} P_{n}\right\|_{p} .
\end{aligned}
$$

We now observe that $\Delta_{\rho}$ and $(-\widetilde{\Delta})^{-1 / 2}$ commute and hence

$$
\begin{gathered}
\omega^{2 l}\left((-\widetilde{\Delta})^{-1 / 2} P_{n}, n^{-1}\right)_{p}=\sup \left\{\left\|\Delta_{\rho}^{2 l}(-\widetilde{\Delta})^{-1 / 2} P_{n}\right\|_{p}: \rho \in S O(d),|\rho-I| \leq 1 / n\right\} \\
\leq \sup \left\{\left\|\Delta_{\tau}(-\widetilde{\Delta})^{-1 / 2} \Delta_{\rho}^{2 l-1} P_{n}\right\|_{p}: \rho, \tau \in S O(d),|\rho-I| \leq 1 / n,|\tau-I| \leq 1 / n\right\} .
\end{gathered}
$$

We set $Q_{n, \rho}=\Delta_{\rho}^{2 l-1} P_{n}$ and note that if $P_{n} \in \operatorname{span}\left(\bigcup_{k=1}^{n-1} H_{k}\right)$, also $Q_{n, \rho} \in \operatorname{span}\left(\bigcup_{k=1}^{n-1} H_{k}\right)$, and using $(2.2)$ as well as Theorems 3.4 and 8.2 , we have, for $|\tau-I| \leq 1 / n$ and $1<p<\infty$,

$$
\left\|\Delta_{\tau}(-\widetilde{\Delta})^{-1 / 2} Q_{n, \rho}\right\|_{p} \leq A n^{-1}\left\|\operatorname{grad}_{\tan }(-\widetilde{\Delta})^{-1 / 2} Q_{n, \rho}\right\|_{p} \leq A_{1} n^{-1}\left\|Q_{n, \rho}\right\|_{p},
$$

which completes the proof of (9.4) and of our theorem.

Theorems 9.1 and 9.2 already yield part of our main result of this paper given in the next theorem.

Theorem 9.3. Suppose $f \in L_{p}\left(S^{d-1}\right), 1<p<\infty, d \geq 3$ and $r=$ $1,2, \ldots$ Then

$$
\omega^{r}(f, \theta)_{p} \approx \widetilde{K}_{r}\left(f, \theta^{r}\right)_{p} .
$$

Proof. Using Theorem 9.2 , we need to show only that

$$
\omega^{r}(f, \theta)_{p} \leq C \widetilde{K}_{r}\left(f, \theta^{r}\right)_{p} .
$$


We use $E_{n}(f)_{p} \leq C_{1} \widetilde{K}_{r}(f, 1 / n)_{p}$ (see [Di-98, Th. 6.1]), the realization of $\widetilde{K}_{r}(f, 1 / n)_{p}$ (see [Di-98, Th. 6.2]), and the relation $\omega^{r}(f, 1 / n)_{p} \approx R_{r}\left(f, 1 / n^{r}\right)_{p}$ in Theorem 8.2 to reduce the proof of $(9.5)$ to

$$
\left\|P_{n}^{(r)}\left(e^{u Q M Q^{-1}} \cdot\right)\right\|_{p} \leq C_{1}\left\|(-\widetilde{\Delta})^{r / 2} P_{n}\right\|_{p}
$$

for $P_{n} \in \operatorname{span}\left(\bigcup_{k=1}^{n-1} H_{k}\right)$ with $C_{1} \equiv C_{1}(r, p)$ independent of $Q \in S O(d)$, $M \in \mathcal{M}, n$ and $P_{n}$.

We define $P_{n, r}=(-\widetilde{\Delta})^{-r / 2} P_{n}$, which is in $\operatorname{span}\left(\bigcup_{k=1}^{n-1} H_{k}\right)$, and we need to show

$$
\left\|P_{n, r}^{(r)}\left(e^{u Q M Q^{-1}} \cdot\right)\right\|_{p} \leq C_{1}\left\|P_{n, 0}\right\|_{p}=C_{1}\left\|P_{n}\right\|_{p} .
$$

We use the identity

$$
(-\widetilde{\Delta})^{\alpha}\left(\frac{\partial}{\partial t} Q_{n}\left(e^{t Q M Q^{-1}} x\right)\right)=\frac{\partial}{\partial t}(-\widetilde{\Delta})^{\alpha} Q_{n}\left(e^{t Q M Q^{-1}} x\right),
$$

which is valid for $\alpha \in \mathbb{R}$ and $Q_{n} \in \operatorname{span}\left(\bigcup_{k=1}^{n-1} H_{k}\right)$ with $\alpha=-1 / 2$ and $Q_{n}(x)=P_{n, r-1}^{(r-1)}(x)$, and derive

$$
\left\|P_{n, r}^{(r)}\left(e^{u Q M Q^{-1}} \cdot\right)\right\|_{p} \leq\left\|\operatorname{grad}_{\tan }(-\widetilde{\Delta})^{-1 / 2} P_{n, r-1}^{(r-1)}\left(e^{u Q M Q^{-1}} \cdot\right)\right\|_{p}
$$

(which, using Theorem 3.1, implies for $1<p<\infty$ )

$$
\leq C(r)\left\|P_{n, r-1}^{(r-1)}\left(e^{u Q M Q^{-1}} \cdot\right)\right\|_{p}
$$

(which, using induction on $r$, implies)

$$
\leq \widetilde{C}(r)\left\|P_{n}\left(e^{u Q M Q^{-1}} \cdot\right)\right\|_{p}=\widetilde{C}(r)\left\|P_{n}\right\|_{p}
$$

10. Application. Having Theorem 9.3, we can improve and extend Theorem 2.2 of [Da-Di-Ti]. We obtain the following sharp Jackson and sharp lower estimate for $\omega^{r}(f, t)_{p}$.

Theorem 10.1. For $f \in L_{p}\left(S^{d-1}\right), 1<p<\infty, d \geq 3, r=1,2, \ldots$ and $s=\max (p, 2)$ one has

$$
t^{r}\left\{\sum_{1 \leq k \leq 1 / t} k^{s r-1} E_{k}(f)_{L_{p}\left(S^{d-1}\right)}^{s}\right\}^{1 / s} \leq C \omega^{r}(f, t)_{p}=C \omega^{r}(f, t)_{L_{p}\left(S^{d-1}\right)}
$$

and

$$
t^{r}\left\{\int_{t}^{1 / 2} \frac{\omega^{m}(f, u)_{p}^{s}}{u^{r s+1}} d u\right\}^{1 / s} \leq C \omega^{r}(f, t)_{p}, \quad m>r .
$$

We note that in [Da-Di-Ti, Th. 2.2], $r$ is restricted to even integers. 
Proof. We simply substitute the result of Theorem 9.3 in Da-Di-Ti, Th. 8.1].

Acknowledgements. The authors would like to thank the referee for his/her thorough reading of our manuscript, generous comments and several valuable suggestions. We would especially like to thank him/her for providing us with a simpler proof of (6.8).

The first author was supported by NSERC grant of Canada G121211001. The second author was supported by NSERC grant of Canada A4816.

Added in proof. The optimality of the range $1<p<\infty$ in Theorems 3.1 and 9.3 was recently established by the second author in an article to appear in Studia Mathematica. That is, $\omega^{m}(f, t)_{p} \approx \widetilde{K}_{m}\left(f, t^{m}\right)_{p}$ does not hold for $p=1$ and $p=\infty$.

\section{References}

[An-As-Ro] G. Andrews, R. Askey and R. Roy, Special Functions, Cambridge Univ. Press, 1999.

[Bo-Cl] A. Bonami et J. L. Clerc, Sommes de Cesàro et multiplicateurs des développements en harmoniques spheriques, Trans. Amer. Math. Soc. 183 (1973), 223-263.

[Br-Da] G. Brown and F. Dai, Approximation of smooth functions on compact twopoint homogeneous spaces, J. Funct. Anal. 220 (2005), 401-423.

[Da-Di-04] F. Dai and Z. Ditzian, Combinations of multivariate averages, J. Approx. Theory 131 (2004), 268-283.

[Da-Di-07] —, -, Cesàro summability and Marchaud inequality, Constr. Approx. 25 (2007), 73-88.

[Da-Di-08] -, -, Jackson inequality for Banach spaces on the sphere, Acta Math. Hungar. 118 (2008), 171-195.

[Da-Di-Ti] F. Dai, Z. Ditzian and S. Tikhonov, Sharp Jackson inequalities, J. Approx. Theory 151 (2008), 86-112.

[Di-98] Z. Ditzian, Fractional derivatives and best approximation, Acta Math. Hungar. 81 (1998), 323-348.

[Di-99] - A modulus of smoothness on the unit sphere, J. Anal. Math. 79 (1999), 189-200.

[Di-04] -, Jackson inequality on the sphere, Acta Math. Hungar. 102 (2004), 1-35.

[Di-07] - A note on equivalence of moduli of smoothness on the sphere, J. Approx. Theory 147 (2007), 125-128.

[Di-Iv] Z. Ditzian and K. G. Ivanov, Strong converse inequalities, J. Anal. Math. 61 (1993), 61-111.

[Di-To] Z. Ditzian and V. Totik, Moduli of Smoothness, Springer, 1987.

[Er] A. Erdélyi et al., Higher Transcendental Functions, Vol. II, Bateman Manuscript Project, McGraw-Hill, 1953.

[Ga] F. R. Gantmakher, The Theory of Matrices, Vol. 1, Chelsea Publ., New York, 1959.

[Mu $\quad$ C. Müller, Analysis of Spherical Symmetries in Euclidean Spaces, Appl. Math. Sci. 129, Springer, New York, 1998. 
[St] E. M. Stein, Harmonic Analysis: Real-Variable Methods, Orthogonality, and Oscillatory Integrals, Princeton Univ. Press, Princeton, NJ, 1993.

[St-We] E. M. Stein and G. Weiss, Introduction to Fourier Analysis on Euclidean Spaces, Princeton Univ. Press, Princeton, NJ, 1971.

[Sz] G. Szegö, Orthogonal Polynomials, Amer. Math. Soc., New York, 1967.

F. Dai, Z. Ditzian

Department of Mathematical

and Statistical Sciences

University of Alberta

Edmonton, Alberta

Canada T6G 2G1

E-mail: dfeng@math.ualberta.ca

zditzian@math.ualberta.ca
Hongwei Huang

School of Mathematical Sciences

Xiamen University 361005, Xiamen, Fujian

China

E-mail: hwhuang@263.net

Received April 23, 2009

Revised version July 18, 2009 